\title{
Topo-Geometric Model MZ: Feeded Objects
}

Andrianarizaka Marc Tiana,

Doctoral School in Engineering and Innovation Science and Technology (ED-STII)

Doctoral Research Team for Cognitive Science and Application (EAD-SCA)

University of Antananarivo, MADAGASCAR

\author{
Robinson Matio, \\ Doctoral School in Engineering and Innovation Science and \\ Technology (ED-STII) \\ Doctoral Research Team for Cognitive Science and \\ Application (EAD-SCA) \\ University of Antananarivo, MADAGASCAR
}

\begin{abstract}
Andriamanohisoa Hery Zo,
Doctoral School in Engineering and Innovation Science and Technology (ED-STII)

Doctoral Research Team for Cognitive Science and Application (EAD-SCA)

University of Antananarivo, MADAGASCAR
\end{abstract}

Summary : The Topo-geometric approach MZ requires notions of both vector geometry and affine geometry, and also uses topology concepts to transform the elementary notions of presenting a linear program problem. These different forms or presentation models of problems related to the Topo-geometric approach MZ are initially presented in this article and will be followed by elementary results demonstrated by the use of the supported objects of the supported model. Thus, this article is the precursor of the determination of redundant constraints by the Topo-geometric model MZ. The energized objects will thus constitute the language of the proofs of the subsequent propositions.

Keywords : Linear programming, mathematical writing of the model, writing of the Topo-geometric model MZ, algorithm.

\section{INTRODUCTION}

This paper constitutes the basis of the Topo-geometric model MZ in search of redundant constraints in a problem of linear programming. This article follows the work on the Topo-Geometric MZ model published in MADA-ETI, ISSN 2220-0673, Vol.2, 2016, www.madarevues.gov.mg which develops the basic concept of Topo-geometric models, and presents the powered objects in search of the redundant constraints of a linear programming problem. All the mathematical object classes will first be presented from the simplest to the most complicated, to model the real concept allowing the decision making. Together with the presentation of each class, the existing operations and relationships will be analyzed; the representation of technical constraints by affine half-spaces is the most important part of this work.

\section{THE MEAN OBJECTIVES}

\subsection{The scalars}

A scalar is any real number. Symbolically, a scalar will be represented with a Latin or Greek or lowercase letter.

Examples: $x, y, z, \ldots, \alpha, \ldots$.

\subsection{Indexes}

It is a positive or null integer that will be attached to an object of a model, as a unique identifier. Symbolically the indexes will be represented by $i, j, k \ldots$

To represent the possible values of an index, the following notions will be used:

$\mathrm{i}=1,2 \ldots \mathrm{n}$ meaning that the index $\mathrm{i}$ varies from 1 to $\mathrm{n}$.

When an objective $\mathrm{x}$ of a model is an index, one writes:

$$
\left(x_{i}\right)_{i=1, \ldots, n}
$$

which is a condensed representation of :

$$
\left(\begin{array}{l}
x_{1} \\
x_{2} \\
x_{i 1}
\end{array}\right):
$$

An object can have two indexes as identifiers. This is the case with matrices, for example.

It is represented by

$$
\left(a_{i j}\right)_{\substack{i=1, \ldots, m \\ j=1, \ldots, m}}
$$

which designates the matrix :

$$
\left(\begin{array}{l}
a_{11} \ldots . . a_{i n} \\
a_{i} \\
a_{m 1} \ldots . a_{m n}
\end{array}\right)
$$

To fully exploit the indexes, the symbol $\sum$ (summation) will also be used.

So, to represent

$$
c_{1} x_{1}+c_{2} x_{2}+\ldots . .+c_{n} x_{n}
$$

it will be simply written:

$$
\sum_{j=1}^{n} c_{j} \cdot x_{j}
$$




\section{THE ELEMENTARY OBJECTS}

\subsection{Decision spaces}

Take again the problems LPP in its known form which consists of finding the unknown-real $x_{1}, x_{2}, \ldots . ., x_{n}$, and maximize:

$$
Z=c_{1} \cdot x_{1}+c_{2} x_{2}+\ldots .+c_{n} x_{n}
$$

$$
\begin{aligned}
& \text { And } \begin{array}{l}
\text { which } \quad \text { satisfy } \\
\text { conditions }:
\end{array} \\
& \left\{\begin{array}{l}
a_{11} x_{1}+a_{i 2} x_{2}+\ldots .+a_{1 n} x_{n} \leq b_{1} \\
a_{21} x_{1}+a_{22} x_{2}+\ldots+a_{2 n} x_{n} \leq b_{2} \\
a_{m 1} x_{1}+a_{m 2} x_{2}+\ldots .+a_{m n} x_{n} \leq b_{n}
\end{array}\right.
\end{aligned}
$$

i) $x_{1} \geq 0, x_{2} \geq 0 . \ldots ., x_{n} \geq 0$

where the coefficients:

$$
\begin{aligned}
& \left(c_{j}\right) j=1, \ldots, n \\
& \left(a_{i j}\right) i=1, \ldots, n j=1, \ldots, n \\
& \left(b_{i}\right) i=1, n
\end{aligned}
$$

Each line of the conditions (3.02) is called a technical constraint.

Conditions (3.03) are called non-negativity constraints.

Definition 3.01: decision-action-point-vectors

We call decision-action any n-tuple :

$\left(\begin{array}{l}x_{1} \\ x_{2} \\ x_{n}\end{array}\right)$ Whose $x_{1}, x_{2}, \ldots, x_{n}$ are elements of

It will be noted later :

$$
\left(x_{j}\right) j=1, \ldots, n
$$

Mathematically, we see that it is an element of $\square^{n}$ relative to a given base. It is also a point in the affine space $\square^{n}$ relative to a given landmark.

An action decision will be represented by an affine point. Which brings us to the decision-action definition?

Notation : an action decision is noted using a capital Greek letter: A, B, X, Y

Definition 3.02: decision marker - action - decision space

We call decision-action benchmark, the positive orthonormal geometric benchmark, noted :

$$
\left(O, U_{1}, U_{2}, \ldots ., U_{n}\right)
$$

related to the aforementioned LPP problem. In this reference, the geometric origin $\mathrm{O}$ has a particular meaning : it represents the decision to do nothing, that is, if

$$
\mathrm{O}=\left(\begin{array}{c}
x_{o 1} \\
x_{o 2} \\
x_{0 n}
\end{array}\right), \quad x_{01}=x_{02}=x_{03}=\ldots .=x_{0 n}=0
$$

To each decision, variable $x_{j}$ corresponds the unit vector $U_{j}$.

The affine space $\square^{n}$, equipped with the reference $\left(O, U_{1}, U_{2}, \ldots ., U_{n}\right)$ is called the decision space relating to the LPP problem to be solved.

Despite this striking resemblance to Euclidean affine geometry, a limiting aspect will be presented from the start.

\subsection{Postulate}

In the topo-geometric theory $\mathrm{MZ}$, the orthonormal affine frame of the decision space action of the problem LPP to be solved is unique.

This assumption means that :

- The concept of basic change does not exist.

- The concept of change of origin does not exist.

However, following a presolved analysis, it may be possible that $\mathrm{n}$ changes, which completely modifies the LPP problem.

\section{OPERATIONS ON ACTION-DECISIONS}

Given an LPP problem, according to definition 3.01, a decision is represented by a point in the decision-action space and / or by a vector of the vector space $\mathbb{R}^{n}$

This dual nature (both affine and vectorial) of decisionactions is very important.

It allows to express :

The creation of other stock-decisions based on existing stock-decisions

The search for other decision-actions according to a given direction.

\subsection{Amplifier of an action decision}

Let the decision-action space relate to the reference.

Let $\mathrm{X}_{0}$ be an action decision and $\alpha$ a given positive real number.

We call amplifier of $\mathrm{X}_{0}$ using $\alpha$, the generation of an action decision $X$ such that

$$
\mathrm{X}=\alpha \mathrm{X}_{0}
$$

Mathematically, it is therefore the multiplier of vector $\mathrm{X}_{0}$ by the scalar $\alpha$

\subsection{Addition of two decision-actions}

Let $\mathrm{X}_{0}$ and $\mathrm{X}_{1}$ be two decision-actions in a LPP problem.

The addition of $\mathrm{X}_{0}$ and $\mathrm{X}_{1}$ is called the creation of a new action decision $\mathrm{X}$ such that

$$
X=X_{0}+X_{1}
$$

Note 4.01

The combination of 2.3.1 and 2.3.2 allows us to model the conic combination concept of two or more action-decisions. Let the decision-actions $\mathrm{X}_{1}, \mathrm{X}_{2} \ldots \mathrm{X}_{\mathrm{k}}$.

The conic combination of these decisions is the new decision $\mathrm{X}$ defined by:

$$
X=\alpha_{1} X_{1}+\alpha_{2} X_{2}+\ldots+\alpha_{k} X_{k}
$$

$\alpha_{1}, \alpha_{2} \ldots ., \alpha_{\mathrm{k}}$ are positive real numbers or zero. 
Note 4.02

From the previous remark, it is clear that the decision satisfies the constraint (2.03) if it is a conic combination of $\mathrm{U}_{1}, \mathrm{U}_{2} \ldots \mathrm{U}_{\mathrm{n}}$, that is to say:

$X=\alpha_{1} U_{1}+\alpha_{2} U_{2}+\ldots+\alpha_{n} U_{n}$ with $\alpha_{1} \geq 0, \alpha_{2} \geq 0 \ldots \alpha_{n} \geq 0$

Let's show that it is reciprocal and true.

Let $\mathrm{X}$ be an action decision satisfying the constraint (2.03)

but

$$
X=\left(x_{j i}\right) \text { with } \quad x_{j i} \geq 0
$$

$$
(x j)=\sum_{j=1}^{n} x_{j} U_{j} \text { with } \mathrm{x}_{\mathrm{j}} \geq 0
$$

4.3 Search axe defined by decision-action Xo and direction the vector decision-action $V$

Let $\mathrm{X}_{0}$ and $\mathrm{V}$ be action-decisions.

We call search axis starting from $X_{o}$ and direction $U$ the set of decision actions, noted $\mathbb{R}\left(\mathrm{X}_{0}, \mathrm{~V}\right)$ defined by

$$
\mathbb{R}\left(\mathrm{X}_{0}, \mathrm{~V}\right)=\left\{\mathrm{X} \in \mathbb{R}^{\mathrm{n}} / \mathrm{X}=\mathrm{X}_{0}+\alpha \mathrm{V} \text {, with } \quad \alpha \in \mathbb{R} \text { and } \alpha \geq 0\right\}
$$

Mathematically, it is the affine ray derived from $\mathrm{X}_{0}$ and the vector $\mathrm{V}$.

Conceptually, the research idea is justified by the fact that in practice, any solver is based on a search algorithm starting from an initial point.

Thus, from a given decision-action $\mathrm{X}_{0}$, and a given search direction $\mathrm{V}$, one can search the points in the universe of decisions that will satisfy the constraints (3.02) and (3.03).

This research idea is not confined to a single research direction. It is also possible to adopt simultaneously two search directions $V_{1}$ and $V_{2}$. Hence the concept of "research plan ", Starting from $\mathrm{X}_{0}$ and direction $\mathrm{V}_{1}$ and $\mathrm{V}_{2}$.

\subsection{Plan of research starting from a decision-action $X_{0}$ and directions $V_{1}$ and $V_{2}$}

Let $\mathrm{X}_{0}$ be a decision-action point and $\mathrm{V}_{1}$ and $\mathrm{V}_{2}$ two decision-action vectors of it.

We call search plane starting from $\mathrm{X}_{0}$ and directions $\mathrm{V}_{1}$ and $\mathrm{V}_{2}$, the set noted $\mathrm{P}\left(\mathrm{X}_{0}, \mathrm{~V}_{1}, \mathrm{~V}_{2}\right)$ defined as follows :

$\mathrm{P}\left(\mathrm{X}_{0}, \mathrm{~V}_{1}, \mathrm{~V}_{2},\right)=\left\{\mathrm{X} \in \mathbb{R}^{\mathrm{n}} / \mathrm{X}=\mathrm{X}_{0}+\alpha_{1} \mathrm{~V}_{1}+\alpha_{2} \mathrm{~V}_{2}\right.$, where $\alpha_{1} \in \mathbb{R}^{+}$and $\left.\alpha_{2} \in \mathbb{R}^{+}\right\}$

By generalizing, we have :

4.5 Research of $\quad X_{0}$ direction $\quad$ and $\quad V_{l}, V_{2 \ldots}$ $V_{k}$, vectors decisions

Let $\mathrm{X}_{0}$ be a decision-action point associated with a LPP.

Let $\mathrm{V}_{1}, \mathrm{~V}_{2} \ldots \mathrm{V}_{\mathrm{k}}, \mathrm{k}$ decision- action vectors of the same problem LPP.

We call vertex cone $\mathrm{X}_{0}$ and directions $\mathrm{V}_{1}, \mathrm{~V}_{2}, \ldots, \mathrm{V}_{\mathrm{k}}$, the action decision set denoted:

$\mathrm{C}\left(\mathrm{X}_{0}, \mathrm{~V}_{1}, \mathrm{~V}_{2} \ldots \mathrm{V}_{\mathrm{k}}\right)$ defined as follows:

$\mathrm{C}\left(\mathrm{X}_{0}, \mathrm{~V} 1, \mathrm{~V}_{2} \ldots \mathrm{V}_{\mathrm{k}}\right)$.

It should be noted at once that research axes and research plans are only special cases of research axes.

\section{THE TECHNICAL CONSTRAINTS}

In practice, any action-decision always has an impact. In the field of linear programming, two categories of impacts can be distinguished :

- Impact-performance.

- Resource impacts.

In this study, we only deal with the impact-resources.

Definition 5.01 resource impact

Let $\mathrm{X}$ be an action decision point of a given LPP problem. Let $\mathrm{V}$ be a null vector of $\square^{n}$.We call resource impact according to $\mathrm{V}$, the scalar product of $\mathrm{X}$ and $\mathrm{V}$ in $\square^{n}$ noted:

$$
\mathfrak{I}(\mathrm{X}, \mathrm{V})=\langle\mathrm{X}, \mathrm{V}\rangle
$$

Where $\langle\mathrm{X}, \mathrm{V}\rangle$ denotes the scalar product $\mathrm{U}$ with $\mathrm{V}$.

The vector $\mathrm{V}$ is called unit consumption vector $\mathrm{V}$ of the given resource.

\section{Definition 5.02 constraint resources}

In our theory, we assume that a constraint that is logical or material may be associated with a resource that is always limited in the associated LPP problem.

Let $A=\left(a_{j}\right)_{j=1 \ldots n}$ be a vector $V$ of consumption of a given resource.

Let $b$ be the limit value of the resource, the zone of respect of the consumption of the resource limited by $b$ is the set noted ZR (A, b) defined by :

$$
\mathrm{ZR}(\mathrm{A}, \mathrm{b})=\left\{X \in \square^{n} / \prec A, X \succ \leq b\right\}
$$

In affine geometry, this set is none other than the negative half-space delimited by the noted affine hyperplane. HP (A, b) defined by:

$$
\mathrm{HP}(\mathrm{A}, \mathrm{b})=\left\{X \in \square^{n} / \prec A, X \succ=b\right\}
$$

NB: The vector A is not an action-decision it is linked to a given resource and allows to calculate the impact of an action $\mathrm{X}$ decision on this resource

6 The constraints of non-negativity

The non-negativity constraints translate the fact that the elementary decisions $\mathrm{x}_{\mathrm{i}}$, where $\mathrm{i}=1 \ldots \mathrm{n}$ must be nonnegatives, is :

$$
x_{i} \geq 0 \quad \forall \mathrm{i}=1,2 \ldots \mathrm{n}
$$

Let us note immediately that the relations :

$$
x_{i} \geq 0 \quad \forall \mathrm{i}=1,2 \ldots \mathrm{n}
$$

can be written:

$$
x_{i} \leq 0 \quad \forall \mathrm{i}=1,2 \ldots \mathrm{n}
$$

Expression equivalent to:

$$
<-U_{i}, X>\leq 0
$$

Therefore, a non-negativity constraint can also be considered as a resource constraint delimited by $Z R=\left(-U_{i}, 0\right)$ called non-negativity zone $Z_{N N}$.

However the combination of all non-negativity constraints has a particular geometric meaning that we call " nonnegativity cone ". 


\section{1 non-negativity cone}

We call non-negativity cone noted $C_{0}^{+}$the cone of search from point-decision $\mathrm{O}$ and direction of all decision vectors :

$$
U_{i}, i=1,2, \ldots, n
$$

Geometrically we have:

$$
\begin{aligned}
C_{0}^{+} & =\left\{X \in \square^{n} / X=\sum_{i=1}^{n} \alpha_{i} U_{i} \text { where } \alpha_{i} \geq 0\right\} \\
& =\text { Cône }\left(O, U_{1}, U_{2}, \ldots, U_{n}\right)
\end{aligned}
$$

Note 6.01:

It is obvious that $\quad C_{o}^{+}=\cap_{i=1}^{i} Z N N_{i}$

\subsection{Non-negativity face}

It is to be recalled that the geometric topo reference of the LPP problem is immutable $\left(\mathrm{O}, \mathrm{U}_{0}, \mathrm{U}_{2} \ldots, \mathrm{U}_{\mathrm{n}}\right)$.

This means that we cannot change the place of a vector $U_{i}$ of this reference, for all $\mathrm{i}$, for all

$\mathrm{i}=1,2 \ldots, \mathrm{n}$.

We call the non-negativity face, denoted by $\mathrm{FNN}_{\mathrm{i}}$, the decision point set defined as follows:

$$
F N N_{i}=\left\{\begin{array}{l}
P\left(O, U_{1}, U n\right) \text { for } i=1 \\
P\left(O, U_{i-1}, U_{i}\right) \text { for } i=2,3, \ldots, n
\end{array}\right\}
$$

\subsection{Non-negativity axes}

For all $\mathrm{i}=1,2 \ldots \mathrm{n}$, we call non-negativity axis, $\mathrm{ANN}_{\mathrm{i}}$, the set of decision point defined as follows :

$$
\mathrm{ANN}_{\mathrm{i}}=\mathrm{R}\left(\mathrm{O}, \mathrm{U}_{\mathrm{i}}\right) \mathrm{i}=1.2 \ldots \mathrm{n}
$$

Note that $\mathrm{ANN}_{\mathrm{i}}$ is none other than the search axis starting from $\mathrm{O}$ and with the direction $\mathrm{U}_{\mathrm{i}}$.

Recall the concept of redundant constraints.

\section{REDUNDANT CONSTRAINTS}

A redundant constraint is a constraint which can be deleted from a system of linear constraints without changing the feasible region or acceptable solution area.

If we look at the next system of $m$ and $n$ constraints of linear inequality, no negative $(m \geq n)$, we can adopt the matrix writing:

$$
A X \leq B, \quad X \geq 0,
$$

But

$$
\mathrm{A} \in R^{m x n}, \quad b \in R^{m}, \mathrm{X} \in R^{n},
$$

And

$$
0 \in R^{n} \text {. }
$$

Let

$$
A T_{i} \leq b_{\mathrm{i}}
$$

be the $i^{\text {th }}$ constraint of system (7.01) and let

$$
S=\left\{\mathrm{X} \in R^{n} / A T_{i} X \leq b_{i}, X \geq 0\right\}
$$

The acceptable solution area associated with the system (7.01).

Let

$$
\mathrm{S}_{k}=\left\{\mathrm{X} \in R^{n} / A T_{i} X \leq b, X \geq 0, \mathrm{i} \neq k\right\}
$$

The acceptable solution area associated to the constraint :

$$
A_{i} X \leq b_{i}, \quad \mathrm{i}=1,2, \ldots, m, i / \neq k
$$

of the system .

The $k^{\text {th }}$ constraint :

$$
\mathrm{A}_{k} X \leq b_{k} \quad(1 \leq k \leq m)
$$

is redundant for the system (7.01) if and only if

$$
\mathrm{S}=\mathrm{S}_{\mathrm{k}} \text {. }
$$

\section{Definition 7.01}

Redondant constraints can be classified as weak and strong redundant constraints. 1.3.2

Low redundancy constraints

The constraint $\mathrm{AT}_{\mathrm{i}} \mathrm{X} \leq \mathrm{b}_{\mathrm{i}}$ is

weakly redundant if she is redundant and

$\mathrm{AT}_{\mathrm{i}} \mathrm{X}=\mathrm{b}_{\mathrm{i}}$ for all $\mathrm{X} \in \mathrm{S}$.

\section{RELATIONSHIP BETWEEN MZ OBJECTS}

The relationships described in this section are the basic ones. More complex relationships will be discussed in the next chapter.

Moreover, the combinations between point-decisions and point-vectors have already been seen. Therefore, only the following points will be dealt with:

- The relation between a decision-point and a constrainttechnical zone.

- The relation between a research axis and a zone of technical constraints.

- The relation between a research plan and a zone of technical constraints.

\subsection{Relationship between a decision--point and a technical constraint zone}

Since a point-decision is represented with a point, and a zone of technical constraints is represented by a half-space, a set of decision-point, the essential same basic relation between one of these two object classes, is the ensemblist relation of belonging.

Let $\mathrm{X}_{0}$ be a decision point and ZCT $(\mathrm{A}, \mathrm{b})$ a constraint zone. The relationship

$$
\mathrm{X}_{\mathrm{o}} \in \mathrm{ZCT}(\mathrm{A}, \mathrm{b}) \text { means }<\mathrm{A}, \mathrm{X}_{\mathrm{o}}>\leq \mathrm{b}
$$

Since in the LPP problem model, $_{2}$ the constraints are indexed, i.e. numbered from 1 to $n$, the corresponding technical constraints areas will also be noted. :

$$
\mathrm{ZCT}_{\mathrm{i}} \quad \mathrm{i}=1,2 \ldots \mathrm{n}
$$

This makes it possible to represent the system of techniques as follows:

Let $\mathrm{X}_{0}$ be a decision-point satisfying all the technical constraints of the problem.

We have :

$$
<\mathrm{A}_{\mathrm{i}}, \mathrm{X}_{0}>\leq \mathrm{b}_{\mathrm{i}} \quad \text { for } \quad \mathrm{i}=1,2, \ldots, \mathrm{n}
$$

This can be written :

$$
X_{0} \in Z C T_{i} \quad \text { For everything } \mathrm{i}=1,2 \ldots \mathrm{n}
$$

From where,

$$
X_{0} \in \cap_{i=1}^{m} Z C T_{i}
$$


Relationship that is a basis for redundancy and infeasibility analysis.

Moreover, when $\mathrm{X}_{0}$ does not belong to a $\mathrm{ZCT}_{\mathrm{i}}$, we also say that $\mathrm{X}_{0}$ is exterior to $\mathrm{ZCT}_{\mathrm{i}}$ as the outer term, and its inner opposite has a topological connotation, the topological definitions of these terms will be recalled.

\subsubsection{Projection of a point on a hyperplane}

Let $\mathrm{ZCT}_{\mathrm{i}}$ be the area of technical constraints delimited by the HP hyperplane $\left(\mathrm{A}_{\mathrm{i}}, \mathrm{b}_{\mathrm{i}}\right)$ and let $\mathrm{X}_{0}$ be any decision-point. We call projection of $\mathrm{X}_{0}$ on the hyperplane $\operatorname{HP}\left(\mathrm{A}_{\mathrm{i}}, \mathrm{b}_{\mathrm{i}}\right)$, the point noted $\mathrm{X}^{\prime}{ }_{0}$ such that :

$\mathrm{X}_{0}$ belongs to $\mathrm{HP}\left(\mathrm{A}_{\mathrm{i}}, \mathrm{b}_{\mathrm{i}}\right)$ and is collinear to the vector $\mathrm{A}_{\mathrm{i}}$. $\mathrm{X}_{0}^{\prime}$ is the intersection of $\mathrm{HP}\left(\mathrm{A}_{\mathrm{i}}, \mathrm{b}_{\mathrm{i}}\right)$ with the straight line passing through $\mathrm{X}_{0}$ and whose direction vector is $\mathrm{A}_{\mathrm{i}}$.

Proof :

Mathematically this right is defined by :

$X \in \square^{n}$ with $X=X_{0}+\alpha A_{i,} \quad \alpha \in \square$

The intersection is written,

$$
\begin{aligned}
& \mathrm{X}_{0}^{\prime}=\mathrm{X}_{0}+\alpha \mathrm{A}_{\mathrm{i}} \\
& <A_{i}, X_{0}^{\prime}>=b_{i} \\
& <A_{i}, X_{0}+\alpha A_{i}>=b_{i} \\
& <A_{i}, X_{0}>+\alpha<A_{i}, A_{i}>=b_{i} \\
& \Rightarrow \alpha<A_{i}, A_{i}>=b_{i}-<A_{i}, X_{0}> \\
& \Rightarrow \alpha=\frac{b_{i}-<A_{i}, X_{0}>}{<A_{i}, A_{i}>}
\end{aligned}
$$

Finally, we have :

$$
X_{0}=X_{0}+\left[\frac{b_{i}-<A_{i}, X_{0}>}{<A_{i}, A_{i}>}\right] A_{i}
$$

Moreover, the distance between $\mathrm{X}_{0}$ and $\mathrm{X}_{0}^{\prime}$ denoted $\mathrm{d}\left(\mathrm{X}_{0}\right.$, $\left.\mathrm{X}_{0}^{\prime}\right)$ is equal to :

$$
\begin{aligned}
& d\left(X_{0}, X_{0}^{\prime}\right)=\left|\frac{b i-<A_{i}, X_{0}>}{<A_{i}, A_{i}>}\right| \sqrt{<A_{i}, A_{i}>} \\
& d\left(X_{0}, X_{0}^{\prime}\right)=\left|\frac{b_{i}-<A_{i}, X_{0}>}{\sqrt{<A_{i}, A_{i}>}}\right|
\end{aligned}
$$

This distance is also called the distance from the point Xo to the hyperplane $\operatorname{HP}\left(\mathrm{A}_{\mathrm{i}}, \mathrm{b}_{\mathrm{i}}\right)$.

\subsubsection{Orientation of the vector Ai with respect to $Z C T_{i}$}

\section{Proposition 8.01}

The vector is always oriented from inside to outside of $\mathrm{ZCT}_{\mathrm{i}}$.

Proof:

Let $\mathrm{X}_{0} \quad$ a $\mathrm{ZCT}_{\mathrm{i}}$ point that does not belong to the hyperplane $\operatorname{HP}\left(\mathrm{A}_{\mathrm{i}}, \mathrm{b}_{\mathrm{i}}\right)$ :

$$
X_{0} \in Z C T_{i} \quad \text { and } \quad X_{0} \notin H P\left(A_{i}, b_{i}\right)
$$

Is $X_{0}{ }^{\prime}$ the projection of $X_{0}$ on $H P\left(A_{i}, b_{i}\right)$

We have :

$$
\begin{gathered}
X_{0}{ }^{\prime}=X_{0}+\left(\frac{b_{i}-<A_{i}, X_{0}>}{<A_{i}, A_{i}>}\right) A_{i} \\
\overrightarrow{X_{0} X_{0}}\left[\frac{b_{i}-<A_{i}, X_{0}>}{<A_{i}, A_{i}>}\right] A_{i}
\end{gathered}
$$

The product of $\overrightarrow{X_{0} X_{0}}$ with $A_{i}$ is :

$$
\begin{aligned}
& <\overrightarrow{X_{0} X_{0}^{\prime}}, A_{i}>=\left(\frac{b_{i}-<A_{i}, X_{0}>}{<A_{i}, A_{i}>}\right)<A_{i}, A_{i}> \\
& <\overrightarrow{X_{0} X_{0}}, A_{i}>=b_{i}-<A_{i}, X_{0}> \\
& \text { but } \quad X_{0} \in Z C T i
\end{aligned}
$$

which means :

$$
<A_{i}, X_{0}>\leq b_{i} \quad \text { and } \quad X_{0} \notin H P\left(A_{i}, b_{i}\right)
$$

So $<A_{i}, X_{0}>\leq b_{i}$

Therefore we have: $<A_{i}, X_{0}><b_{i}$

From where $\quad b_{i}-<A_{i}, X_{0}>>0$

Finally we get the following relation:

$$
<\overrightarrow{X_{0} X_{0}{ }^{\prime}}, A_{i}>>0
$$

$\checkmark 8.1 .3$

Direction of a decision vector with respect to $Z C T_{i}$

Let $\mathrm{V}$ be a decision vector, and let $\mathrm{ZCT}_{\mathrm{i}}$ be a technical constraint zone $\mathrm{V}$ parallel to $\operatorname{HP}\left(\mathrm{A}_{\mathrm{i}} ; \mathrm{b}_{\mathrm{i}}\right)$, that is to say at the $\mathrm{ZCT}_{\mathrm{i}}$ boundary.

\subsubsection{External orientation}

We say that $\mathrm{V}$ has an external orientation with respect to $\mathrm{ZCT}_{\mathrm{i}}$, if $<\mathrm{V}, \mathrm{A}_{\mathrm{i}}>$ is strictly positive.

\subsubsection{Parallel orientation}

We say that $\mathrm{V}$ has a parallel orientation with respect to $\mathrm{ZCT}_{\mathrm{i}}$, if $\left\langle\mathrm{V}, \mathrm{A}_{\mathrm{i}}\right\rangle$ is equal to 0 .

Note 8.01:

The qualification "parallel" refers to the fact that $\mathrm{V}$ is parallel to $\mathrm{HP}\left(\mathrm{A}_{\mathrm{i}} ; \mathrm{b}_{\mathrm{i}}\right)$, that is to say at the $\mathrm{ZCT}_{\mathrm{i}}$ border.

\subsubsection{Internal orientation}

We say that $\mathrm{V}$ has an internal orientation with respect to $\mathrm{ZCT}_{\mathrm{i}}$, if $\left\langle\mathrm{V}, \mathrm{A}_{\mathrm{i}}\right\rangle$ is strictly negative.

\subsubsection{Characterization of an Inner Point of $Z C T_{1}$ \\ Proposition 8.02}

$\mathrm{X}_{0}$ is an inner point of $\mathrm{ZCT}_{\mathrm{i}}$ if and only if :

Proof:

$$
<A_{i}, X_{0}><b_{i}
$$

a) Let $\mathrm{X}_{0}$ be a point inside $\mathrm{ZCT}_{\mathrm{i}}$.

According to 2.7.1.3.1, there exists a strictly positive reality $\mathrm{r}$ such that the ball whose center is $\mathrm{X}_{0}$ and with a radius $r$ is entirely contained in $\mathrm{ZCT}_{\mathrm{i}}$. 
Logically, this means that if $\mathrm{X}_{0}{ }^{\prime}$ designates the projection of $\mathrm{X}_{0}$ on $H P\left(A_{i}, b_{i}\right)$, then the distance between $\mathrm{X}_{0}$ and $\mathrm{X}_{0}{ }^{\prime}$ is equal to :

$$
\frac{\left|b_{i}-<A_{i}, X_{0}>\right|}{\sqrt{<A_{i}, A_{i}>}}
$$

And

$\mathrm{r} \leq \frac{\mathrm{b}_{\mathrm{i}}<\mathrm{A}_{\mathrm{i}}, \mathrm{X}_{0}>}{\sqrt{<\mathrm{A}_{\mathrm{i}}, \mathrm{A}_{\mathrm{i}}>}}$ but $\mathrm{r}$ is strictly positive,

$\frac{\mathrm{b}_{\mathrm{i}}-<\mathrm{A}_{\mathrm{i}}, \mathrm{X}_{0}>}{\sqrt{<\mathrm{A}_{\mathrm{i}}, \mathrm{A}_{\mathrm{i}}>}}$ is strictly positive from where

$\mathrm{b}_{\mathrm{i}}-<\mathrm{A}_{\mathrm{i}}, \mathrm{X}_{0}>$ is alsostrictly positive.

b) Reciprocally, $\mathrm{X}_{0}$ is a point of $\mathrm{ZCT}_{\mathrm{i}}$ such that $<A_{i}, X_{0}><b_{i}$

Let us show that this is an inside point of $\mathrm{ZCT}_{\mathrm{i}}$.

Let $\mathrm{X}_{0}{ }^{\prime}$ be the projection of $\mathrm{X}_{0}$ on the hyperplane. We saw that :

The distance between $\mathrm{X}_{0}$ and $\mathrm{X}_{0}{ }^{\prime}$ is equal to:

$$
\frac{b_{i}-<A_{i}, X_{0}>}{\sqrt{<A_{i}, A_{i}>}}
$$

Let

$$
r=\frac{1}{2} \frac{b_{i}-<A_{i}, X_{0}>}{<A_{i}, A_{i}>}
$$

We have $\quad r>0$;

and it is obvious that the ball whose center is $\mathrm{X}_{0}$ and radius $r$ is entirely included in $Z^{2} T_{i}$, which means that $X_{0}$ is an internal point of $\mathrm{ZCT}_{\mathrm{i}}$.

\subsubsection{S border point}

A point $\mathrm{X}_{0} \in \square^{n}$ is said to be a border point of $\mathrm{S}$ if :

$\mathrm{X}_{0}$ is an element of Set ;

Whatever the positive real number $r$ is, the open ball of center $\mathrm{X}_{0}$ and radius $\mathrm{r}$ contains both elements of $\mathrm{S}$ other than $\mathrm{X}_{0}$ and elements of $\bar{S}$ (the complement of $\mathrm{S}$ in Euclidean affine space $\square^{n}$ ).

\subsection{5 $\mathrm{S}$ border}

Let $\mathrm{S}$ be a non-empty set in the Euclidean affine space with the orthonormal coordinate system

$\left(\mathrm{O}, U_{1}, U_{2}, \ldots, U_{n}\right)$. We call the boundary of $\mathrm{S}$ the set noted

Fr (S) containing all the boundary points of $S$.

\section{Proposition 8.03}

We characterize the border points of the $\mathrm{ZCT}_{\mathrm{i}}$ by:

$$
\operatorname{Fr}\left(\mathrm{ZCT}_{\mathrm{i}}\right)=\operatorname{HP}\left(\mathrm{A}_{\mathrm{i}} ; \mathrm{b}_{\mathrm{i}}\right)
$$

\section{Proof:}

Let's first show the first inclusion :

$$
\operatorname{Fr}\left(Z C T_{i}\right) \subset H P\left(A_{i}, b_{i}\right)
$$

Let $\mathrm{X}_{\mathrm{o}}$ be an element of $\operatorname{Fr}\left(\mathrm{ZCT}_{\mathrm{i}}\right)$, that means that $\mathrm{X}_{0}$ is also an element of $\mathrm{ZCT}_{\mathrm{i}}$ so,

$$
<A_{i}, X_{0}>\leq b_{i}
$$

If $X o \notin H P\left(A_{i}, b_{i}\right)$, this means that $<A_{i}, X_{0}>\neq b_{i}$

From where,

$<A_{i}, X_{0}><b_{i}$, meaning that $\mathrm{X}_{0}$ is an inner point of $\mathrm{ZCT}_{\mathrm{i}}$, which contradicts the fact that $\mathrm{X}_{0}$ is a border point of $\mathrm{ZCT}_{\mathrm{i}}$.

Reciprocal :

$$
H P\left(A_{i}, b_{i}\right) \subset \operatorname{Fr}\left(Z C T_{i}\right)
$$

Let $\mathrm{X}_{0}$ be a point of HP $\left(\mathrm{A}_{\mathrm{i}}, \mathrm{bi}\right)$ which means that :

$$
<A_{i}, X_{0}>=b_{i}
$$

Let $r$ be any strictly positive real number and $X_{1}$ the point defined by :

$$
X_{1}=X_{0}+\frac{r}{2} \cdot \frac{A_{i}}{\sqrt{<A_{i}, A_{i}>}}
$$

Note that the distance between $\mathrm{X}_{0}$ and $\mathrm{X}_{1}$ is equal to $\frac{r}{2}$, which is strictly less than $r$.

Moreover, according to Proposition 8.01, $\mathrm{A}_{\mathrm{i}}$ is always oriented towards the outside of $\mathrm{ZCT}_{\mathrm{i}}$ so $\mathrm{X}_{1}$ do not belong to $\mathrm{ZCT}_{\mathrm{i}}$.

So $\mathrm{X}_{1}$ belongs to the open ball of center $\mathrm{X}_{0}$ and radius $\mathrm{r}$.

Finally, let us show that the open ball of center $\mathrm{X}_{0}$ and radius $\mathrm{r}$ contains points of $\mathrm{ZCT}_{\mathrm{i}}$ other than $\mathrm{X}_{0}$.

Let $\mathrm{X}_{2}$ the point defined by :

$$
X_{2}=X_{0}-\frac{r}{2} \cdot \frac{A_{i}}{\sqrt{\left.<A_{i}, A_{i}\right\rangle}}
$$

We obtain:

$$
\begin{aligned}
<A_{i}, X_{2}> & =<A_{i}, X_{0}-\frac{r}{2} \cdot \frac{A_{i}}{\sqrt{<A_{i}, A_{i}}}> \\
& =<A_{i}, X_{0}>-\frac{r}{2} \cdot \frac{1}{\sqrt{<A_{i}, A_{i}>}}<A_{i}, A_{i}> \\
<A_{i}, X_{2}> & =<A_{i}, X_{0}>-\frac{r}{2}
\end{aligned}
$$

But

$$
<A_{i}, X_{0}>=b_{i} \Rightarrow \quad<A_{i}, X_{2}>=b_{i}-\frac{r}{2}
$$

As

$$
r>0 \Rightarrow \frac{r}{2}>0
$$

From where

$$
b_{i}-\frac{r}{2}<b_{i}
$$

We can write :

$$
<A_{i}, X_{2}><b_{i}
$$


Which means that $X_{2}$ belongs to $\mathrm{ZCT}_{\mathrm{i}}$.

In summary, every strictly positive real number in the open ball of center $\mathrm{X}_{0}$ and radius $\mathrm{r}$ contains both elements of $\mathrm{ZCT}_{\mathrm{i}}$ other than $\mathrm{X}_{0}$ and elements of $\mathrm{ZCT}_{\mathrm{i}}$.

This means that $\mathrm{X}_{0}$ is a frontier point of $\mathrm{ZCT}_{\mathrm{i}}$.

Finally, in combination with the two way, we have :

$$
\begin{aligned}
& \operatorname{Fr}\left(Z C T_{i}\right) \subset \operatorname{HP}\left(A_{i}, b_{i}\right) \\
& \operatorname{Fr}\left(Z C T_{i}\right) \supset \operatorname{HP}\left(A_{i}, b_{i}\right)
\end{aligned}
$$

So we have

$$
\operatorname{Fr}\left(Z C T_{i}\right)=\operatorname{HP}\left(A_{i}, b_{i}\right)
$$

\section{Proposition 8.04}

The zone of technical constraints $\mathrm{ZCT}_{\mathrm{i}}$ is topologically closed.

Proof :

Let $\mathrm{X}_{0}$ be a point not belonging to $\mathrm{ZCT}_{\mathrm{i}}$ (where $\mathrm{ZCT}_{\mathrm{i}}$ denotes the complement of $\mathrm{ZCT}_{\mathrm{i}}$ in the affine space).

As

$$
X_{0} \notin Z C T_{i}
$$

So

$$
<A_{i}, X_{0}>>b_{i}
$$

Let $\mathrm{X}_{0}$ ' be the projection of $\mathrm{X}_{0}$ on $\operatorname{HP}\left(A_{i}, b_{i}\right)$. We showed that :

The distance between $\mathrm{X}_{0}$ ' and $\mathrm{X}_{0}$ is equal to :

$$
\frac{\left|b_{i}-<A_{i} \quad X_{0}>\right|}{\sqrt{A_{i}, A_{i}}} .
$$

but

$$
<A_{i}, X_{0}>>b_{i} \Rightarrow b_{i}-<A_{i}, X_{0}><0
$$

so

$$
\begin{aligned}
\left|b_{i}-<A_{i}, X_{0}>\right|= & -\left(b_{i}-<A_{i}, X_{0}>\right) \\
= & <A_{i}, X_{0}>-b_{i}
\end{aligned}
$$

The distance between $\mathrm{X}_{0}$ and the border $\operatorname{HP}\left(A_{i}, b_{i}\right)$ is equal to :

$$
\frac{<A_{i}, X_{0}>-b_{i}}{\sqrt{<A_{i}, A_{i}>}}
$$

Let :

$$
r=\frac{1}{2} \cdot \frac{<A_{i}, X_{0}>-b_{i}}{\sqrt{<A_{i}, A_{i}>}} \text { be }
$$

It is easy to show that the open ball of center $\mathrm{X}_{0}$ and radius $\mathrm{r}$ is entirely contained in $\overline{Z C T_{i}}$.

Therefore $\overline{Z C T_{i}}$ is open.

So $\mathrm{ZCT}_{\mathrm{i}}$ is closed.

In summary of Proposition 8.03 and Proposition 8.04, each zone of $\mathrm{ZCT}_{\mathrm{i}}$ technical constraints is closed and their boundary is none other than the hyperplane $\operatorname{HP}\left(A_{i}, b_{i}\right)$.
8.2 Relationship between a search axis $R\left(X_{0}, V\right)$ and $a$ technical constraint zone $Z C T_{i}$

Consider an area of $\mathrm{ZCT}_{\mathrm{i}}$ technical constraints. Let also be the radius $\mathrm{R}\left(\mathrm{X}_{0}, \mathrm{~V}\right)$ coming from $\mathrm{X}_{0}$ and direction vector $\mathrm{V}$, representing a search axis. This relationship is based on the existence and uniqueness of the solution of the equation :

$$
\alpha\left\langle A_{i}, V\right\rangle=b_{i}-\left\langle A_{i}, X_{0}\right\rangle, \alpha \text { is the unknown. }
$$

Proof:

Since the two objects are sets of decision points, the main combination that can be imagined between them is the intersection. In addition, since the area of technical constraint is closed and the hyperplathe $\operatorname{HP}\left(\mathrm{A}_{\mathrm{i}}, \mathrm{b}_{\mathrm{i}}\right)$ is closed, we will be much more interested in the intersection of the radius $\mathrm{R}\left(\mathrm{X}_{0}, \mathrm{~V}\right)$ with this boundary $\mathrm{HP}\left(\mathrm{A}_{\mathrm{i}}, \mathrm{b}_{\mathrm{i}}\right)$. Indeed, if $\mathrm{X}_{0}$ is outside of $\mathrm{ZCT}_{\mathrm{i}}$, then such an intersection gives us the point of entry from the outside to the inside of $\mathrm{ZCT}_{\mathrm{i}}$ along the radius. On the other hand, if $\mathrm{X}_{0}$ is inside $\mathrm{ZCT}_{\mathrm{i}}$, it gives the exit point of $\mathrm{ZCT}_{i}$.

Let I denote this point of intersection : as I belongs to $\mathrm{R}$ $\left(\mathrm{X}_{0}, \mathrm{~V}\right)$

We have $I=X_{0}+\alpha V \quad$ avec $\quad \alpha>0$

Since I also belongs to $\operatorname{HP}\left(\mathrm{A}_{\mathrm{i}}, \mathrm{b}_{\mathrm{i}}\right)$, we can write :

$$
\left\langle A_{i}, I\right\rangle=b_{i}
$$

By combining these two relationships, we have

$$
\left\langle A_{i}, X_{0}+\alpha V\right\rangle=b_{i}
$$

By developing, we get

$$
\left\langle A_{i}, X_{0}\right\rangle+\alpha\left\langle A_{i}, V\right\rangle=b_{i}
$$

which is an equation where $\alpha$ is the unknown.

This equation gives :

$$
\alpha\left\langle A_{i}, V\right\rangle=b_{i}-\left\langle A_{i}, X_{0}\right\rangle
$$

The existence and uniqueness of $\alpha$ depend on the values of $\mathrm{b}$ i - $\left(\mathrm{A}_{\mathrm{i}}, \mathrm{X} \mathrm{o}\right)$ and $\left(\mathrm{A}_{\mathrm{i}}, \mathrm{V}\right)$, hence $\mathrm{X}_{0}$ and $\mathrm{V}$.

\subsubsection{Where $X_{0}$ is outside $Z C T_{i}$ and where $V$ is not facing} inwards from $Z C T_{i}$

The equation :

$$
\alpha\left\langle A_{i}, V\right\rangle=b_{i}-\left\langle A_{i}, X_{0}\right\rangle
$$

has no solution.

Proof:

This case is mathematically translated by :

$$
\left\{\begin{array}{l}
\left.\left\langle A_{i}, X_{0}\right\rangle\right\rangle b_{i} \\
\left\langle A_{i}, V\right\rangle \geq 0
\end{array} \text { and }\left\langle A_{i}, X_{0}\right\rangle\right\rangle b_{i}
$$

leads that,

$$
b_{i}-\left\langle A_{i}, X_{0}\right\rangle\langle 0
$$

Also as

$$
\alpha \geq 0 \quad \text { et } \quad\left\langle A_{i}, V\right\rangle=b_{i}-\left\langle A_{i}, X_{0}\right\rangle\langle O
$$


which is impossible.

In this case I do not exist.

8.2.2 Where $X_{0}$ is outside $Z C T_{i}$ and where $V$ is inward $Z C T_{i}$ The equation :

$$
\alpha\left\langle A_{i}, V\right\rangle=b_{i}-\left\langle A_{i}, X_{0}\right\rangle
$$

has a unique solution.

$$
\left.\alpha=\frac{b_{i}-\left\langle A_{i}, X_{0}\right\rangle}{\left\langle A_{i}, V\right\rangle}\right\rangle 0
$$

Proof:

We have :

$$
b_{i}-\left\langle A_{i}, X_{\mathrm{O}}\right\rangle\left\langle\mathrm{O} \text { et }\left\langle A_{i}, V\right\rangle\langle\mathrm{O}\right.
$$

which gives

$$
\left.\alpha=\frac{b_{i}-\left\langle A_{i}, X_{0}\right\rangle}{\left\langle A_{i}, V\right\rangle}\right\rangle 0
$$

which is in addition a unique value.

In this case we say that we have a single point of entry starting from $\mathrm{X}_{0}$, and moving along the axis of $\mathrm{R}\left(\mathrm{X}_{0}, \mathrm{~V}\right)$.

8.2.3 Where $X_{0}$ is on $Z C T_{i}$ and where $V$ is outside $Z C T_{i}$

The equation :

$$
\alpha\left\langle A_{i}, V\right\rangle=b_{i}-\left\langle A_{i}, X_{0}\right\rangle
$$

admits a null solution.

Proof:

This case results in :

$b_{i}-\left\langle A_{i}, X_{0}\right\rangle=0$ and $\left.\left\langle A_{i}, V\right\rangle\right\rangle \mathrm{O}$

The only solution available is :

$$
\alpha=0
$$

Meaning that,

$$
\mathrm{I}=\mathrm{X}_{0}
$$

8.2.4 Where $X_{0}$ is on the $Z C T_{i}$ border and where $V$ is parallel oriented to

$H P\left(A_{i}, b_{i}\right)$

The equation :

$$
\alpha\left\langle A_{i}, V\right\rangle=b_{i}-\left\langle A_{i}, X_{0}\right\rangle
$$

has an infinity of solutions.

Proof :

Mathematically we translate this case by :

$$
b_{i}-\left\langle A_{i}, X_{0}\right\rangle=0 \text { and }\left\langle A_{i}, V\right\rangle=0
$$

This gives us infinity of solutions. In fact, $\quad \mathrm{R}\left(\mathrm{X}_{0}, \mathrm{~V}\right)$ is included in $\mathrm{HP}\left(\mathrm{A}_{\mathrm{i}} ; \mathrm{b}_{\mathrm{i}}\right)$, and the intersection is none other than $\mathrm{R}\left(\mathrm{X}_{0}, \mathrm{~V}\right)$.
8.2.5 Where $X_{0}$ is on the $Z C T_{i}$ border and where $V$ is inward $Z C T_{i}$

The equation :

$$
\alpha\left\langle A_{i}, V\right\rangle=b_{i}-\left\langle A_{i}, X_{0}\right\rangle
$$

has a unique solution.

Proof :

In this case

$$
b_{i}-\left\langle A_{i}, X_{0}\right\rangle=0 \quad \text { et }\left\langle A_{i}, V\right\rangle\langle 0
$$

which leads to

$$
\alpha=0
$$

That is to say $\mathrm{I}=\mathrm{X}_{0}$, unique solution.

8.2.6 Where $X_{0}$ is inside $Z C T_{i}$ and where $V$ is outside $Z C T_{i}$

The equation :

$$
\alpha\left\langle A_{i}, V\right\rangle=b_{i}-\left\langle A_{i}, X_{0}\right\rangle
$$

has a unique solution

$$
\alpha=\frac{b_{i}-\left\langle A_{i}, X_{\mathrm{o}}\right\rangle}{\left\langle A_{i}, V\right\rangle}
$$

Proof :

This case results

in $\left\langle A_{i}, X_{\mathrm{O}}\right\rangle\left\langle\boldsymbol{b}_{i}\right.$ et $\left.\left\langle\boldsymbol{A}_{i}, V\right\rangle\right\rangle \mathrm{O}$ :

Which leads to:

$$
\left.b_{i}-\left\langle A_{i}, X_{0}\right\rangle\right\rangle 0
$$

Hence the unique solution

$$
\alpha=\frac{b_{i}-\left\langle A_{i}, X_{0}\right\rangle}{\left\langle A_{i}, V\right\rangle}
$$

8.2.7 Case where Xo is inside $Z C T_{i}$ and where $V$ is parallel oriented to $Z C T_{i}$

The equation :

$$
\alpha\left\langle A_{i}, V\right\rangle=b_{i}-\left\langle A_{i}, X_{0}\right\rangle
$$

has no solution.

Proof :

As in the previous case, we have :

$$
\left.b_{i}-\left\langle A_{i}, X_{\mathrm{o}}\right\rangle\right\rangle \text { Obut }\left\langle A_{i}, V\right\rangle=0
$$

Which leads to an impossibility, meaning that $R\left(X_{0}, V\right)$ will never intercept the border of $Z C T_{i}$.

8.2.8 Where $X_{0}$ is inside $Z C T_{i}$ and where $V$ is inward $Z C T_{i}$ The equation :

$$
\alpha\left\langle A_{i}, V\right\rangle=b_{i}-\left\langle A_{i}, X_{0}\right\rangle
$$

has no solution. 
Proof :

As recently,

$\left.b_{i}-\left\langle A_{i}, \quad X_{\mathrm{o}}\right\rangle\right\rangle \mathrm{O}$ and $\left.\left\langle A_{i}, V\right\rangle\right\rangle \mathrm{O}$

In this case, the equation

$$
\alpha\left\langle A_{i}, V\right\rangle=b_{i}-\left\langle A_{i}, X_{0}\right\rangle
$$

has no positive solution, meaning that $R\left(X_{0}, V\right)$ will never intercept the border of $Z C T_{i}$.

8.2. 9 Algorithm for determining the intersection of a search axis $R\left(X_{0}, V\right)$ with the technical constraint zone $Z C T_{i}$

The previous eight cases can be summarized in the following algorithm :

- Case where there is no intersection. No intersection :

- Case with

$$
\left.\left\langle A_{i}, X_{0}\right\rangle\right\rangle b_{i} \quad \text { and }\left\langle A_{i}, V\right\rangle\langle 0
$$

We have a single point of intersection

$$
\left\{X_{0}+\frac{b_{i}-\left\langle A_{i}, X_{0}\right\rangle}{\left\langle A_{i}, V\right\rangle} V\right\}
$$

- If $\left\langle A_{i}, X_{0}\right\rangle=b_{i}$ and $\left.\left\langle A_{i}, V\right\rangle\right\rangle 0$.

There is unique solution :

$$
\left\{X_{0}\right\}
$$

-If $\left\langle A_{i}, X_{0}\right\rangle=b_{i}$ and $\left\langle A_{i}, V\right\rangle\langle 0$.

There is unique solution:

$$
\left\{X_{0}+\frac{b_{i}-\left\langle A_{i}, X_{0}\right\rangle}{A_{i}, V} V\right\}
$$

-If $\left\langle A_{i}, X_{0}\right\rangle\left\langle b_{i} \quad\right.$ and $\quad\left\langle A_{i}, V\right\rangle \geq 0$

No solution : $\varnothing$

8.3 Relationship between a search-plane $C\left(X_{0}, V_{1}\right.$, $V_{2}$ ) with linearly independent to $\quad V_{I}$ and $V_{2}$ and $a$ of $Z C T_{i}$ technical constraints zone

As for section 8.2, these two objects are sets of decision points, so this section will describe their intersection.

Let $\mathrm{I}$ be such a point. It is thus of the form :

$$
I=X_{0}+\alpha_{1} V_{1}+\alpha_{2} V_{2} \text { with } \alpha_{1} \text { and } \alpha_{2} \text { are positives. }
$$

More like I belongs to the border of $Z C T_{i}$, we can write :

$$
\left\langle A_{i}, I\right\rangle=b_{i}
$$

Which leads to :

$$
\left\langle A_{i}, X_{0}+\alpha_{1} V_{1}+\alpha_{2} V_{2}\right\rangle=b_{i}
$$

From where

$$
\left.\left.\left\langle A_{i}, X_{0}\right\rangle+\alpha_{1}\left(A_{i}, V_{1}\right)\right\rangle+\alpha_{2}\left(A_{i}, V_{2}\right)\right\rangle=b_{i}
$$

Let

$$
\alpha_{1}\left\langle A_{1}, V_{1}\right\rangle+\alpha_{2}\left\langle A_{i}, V_{2}\right\rangle=b_{i}-\left\langle A_{i}, X_{0}\right\rangle
$$

which is a linear equation with two unknowns variables, $\alpha_{1}$ et $\alpha_{2}$ positive.
As for 2.6.2, the existence and uniqueness of the solutions to this problem depend on the three objects $X_{0}, V_{1}$ et $V_{2}$.

- For $X_{0}$, there are three possible situations : to be outside of $\mathrm{ZCT}_{\mathrm{i}}$, or be on the border of $\mathrm{ZCT}_{\mathrm{i}}$ or be inside of $\mathrm{ZCT}_{\mathrm{i}}$.

- For $V_{1}$ there are three possibilities : be outward facing from $Z C T_{i}$ or be oriented parallel to $Z C T_{i}$ or be oriented towards the inside of $Z C T_{i}$

- For $\mathrm{V}_{2}$ the possibilities are the same as for $\mathrm{V}_{1}$.

All in all, we have $3 \times 3 \times 3$, that is to say, 27 possible cases to be made.

\subsection{Search along an axis $R\left(X_{0}, V\right)$ in the ZNN non negativity} zone

The ZNN non-negativity zone has been defined as

$$
Z N N=\bigcap_{j=1}^{n} Z N N_{i}
$$

where

$$
Z N N_{i}=Z R\left(-U_{i}, \varnothing\right)
$$

Conceptually, this means that we assimilate $Z N N_{i}$ to a resource constraint zone, and if a $\mathrm{X}_{0}$ point belongs to $Z N N_{i}$ this means that $\mathrm{X}_{0}$ satisfies the constraint associated with $Z N N_{i}$. It is therefore a stage situation.

The concept of research implies that there is a situation or state of deposition, and that from this situation, one move to another situation or state. And we have already seen that this research, when it is linear, can be modeled by the concept of axis of research starting from a given point and moving according to a given vector $R\left(X_{0}, V\right)$

In this section, the starting point of the search is the point $\mathrm{X}_{0}$, which is supposed to be located in the $\mathrm{ZNN}$ nonnegativity zone. Since the new decisions found must have remained in $\mathrm{ZNN}$, it is logical to study the conditions under which this search leads us out of ZNN. And as in the case of $\mathrm{ZCT}_{\mathrm{i}}$ technical constraint zones, we will need to define the boundary concept in $\mathrm{ZNN}$.

For $\mathrm{ZNN}_{\mathrm{i}}$, there is no problem.

Indeed,

$$
\operatorname{Fr}\left(Z N N_{i}\right)=\mathrm{HP}\left(-U_{i}, 0\right)
$$

given that $Z N N_{i}$ is only half affine delimited by the hyperplane $\mathrm{H} P\left(-U_{i}, O\right)$.

And as HP $\left(-\mathrm{U}_{\mathrm{i}}, 0\right)$ is included in $Z N N_{i}$, we know that $\mathrm{ZNN}_{\mathrm{i}}$ is a closed area.

8.4.1 Frontier of the $Z \mathrm{ZN}_{i}$ non-negativity zone Proposition 8.05

$$
\operatorname{Fr}(Z N N)=\bigcup_{j=1}^{n}\left(H P\left(-U_{j}, 0\right) \cap C_{0}^{+}\right)
$$


Proof :

It suffices to show that for every $\mathrm{j}, H P\left(-U_{j}, 0\right) \cap C_{0}^{+}$is a function of ZNN.

\section{Step 1:}

Let us show that every $\mathrm{X}$ point of $H P\left(-U_{j}, 0\right) \cap C_{0}^{+}$is a border point of $\mathrm{ZNN}$.

Let $r$ be any positive real number. This is to show that the open ball of center $\mathrm{X}$ and radius $\mathrm{r}$ denoted $\mathrm{B}(\mathrm{X}, \mathrm{r})$, contains both an element of $\mathrm{ZNN}$ other than $\mathrm{X}$ and an element of ZNN other than $\mathrm{X}$ is:

$$
\mathrm{X}=\left(\mathrm{X}_{\mathrm{i}}\right)_{\mathrm{i}=1 \ldots, \mathrm{n}}
$$

The fact that $X \in \mathrm{HP}\left(-\mathrm{U}_{\mathrm{j}}, \mathrm{o}\right) \cap \mathrm{C}_{\mathrm{o}}$ means that

$$
\mathrm{x}_{\mathrm{i}} \geq 0, \quad \forall \mathrm{i}=1 \ldots \mathrm{n} \text { and that } \mathrm{x}_{\mathrm{j}} \geq 0
$$

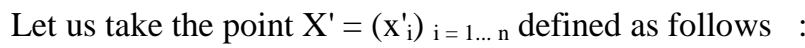

$$
\mathrm{X}_{\mathrm{i}}^{\prime}=\left\{\begin{array}{rc}
x_{i} & \text { for } \text { all } i \neq j \\
-\frac{1}{2} & \text { if } \quad i=j
\end{array}\right.
$$

Similarly let us take the point:

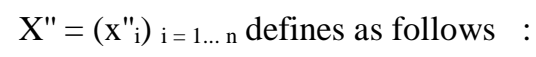

$$
\mathrm{x}_{\mathrm{i}}= \begin{cases}x_{i} & \text { forall } \quad i \neq j \\ +\frac{1}{2} & \text { if } \quad i=j\end{cases}
$$

It is obvious that :

$$
\mathrm{X}^{\prime} \in \mathrm{ZNN} \text { and has } \mathrm{X}^{\prime \prime} \in \mathrm{ZNN}
$$

Moreover, it is also obvious that :

$$
\mathrm{X}^{\prime} \in \mathrm{B}(\mathrm{X}, \mathrm{r}) \text { and } \mathrm{X}^{\prime \prime} \in \mathrm{B}(\mathrm{X}, \mathrm{r})
$$

This shows that the open ball $\mathrm{B}(\mathrm{X}, \mathrm{r})$ contains both the point $\mathrm{X}$ 'which is the element outside ZNN and the point $\mathrm{X}$ " which is the element in $\mathrm{ZNN}$, and it is obvious that $\mathrm{X}^{\prime}$ is different from $X$ and that $X^{\prime \prime}$ is different from $X$.

Therefore $\mathrm{X}$ is a border point of $\mathrm{ZNN}$.

\section{2nd step:}

Let us show that $\mathrm{HP}\left(-\mathrm{U}_{\mathrm{j}}, 0\right) \cap C_{0}^{+}$

And in summary, HP $\left(-\mathrm{U}_{\mathrm{j}}, 0\right)$ is indeed a $\mathrm{ZNN}$ border and their meeting is also a border of $\mathrm{ZNN}$.

In addition, let $\mathrm{X}$ be a point of $\mathrm{ZNN}$ such that :

$$
X \notin \bigcup_{j=1}^{n} H P(-U j, 0) \cap C_{0}^{+}
$$

It means that :

$$
\left.X \notin \bigcup_{j=1}^{n} H P(-U j, 0) \cap C_{0}^{+}\right)
$$

Given that $C_{0}^{+}=\mathrm{ZNN}$, it means that

$$
X \notin \bigcup_{j=1}^{n} H P\left(-U_{j}, 0\right) \cap C_{0}^{+}
$$

Let $X^{j}$ be the projection of $\mathrm{X}$ on $\mathrm{HP}\left(-\mathrm{U}_{\mathrm{j}}, 0\right)$ and $\mathrm{d}_{\mathrm{j}}$ the distance between $X$ and $X_{j}$.

As

$$
X^{j} \neq X \quad \forall_{j}=1, \ldots, n
$$

Then $\mathrm{d}_{\mathrm{j}} \neq \mathrm{o} \quad \forall{ }_{j}=1, \ldots, n$

Let

$$
r=\frac{1}{2} \min (d j) \quad \forall j=1, \ldots, n
$$

It is obvious that $r>0$ and that the open ball $\mathrm{B}(\mathrm{X}, \mathrm{r})$ is included in $\mathrm{ZNN}$.

There fore,

$$
\left.\bigcup_{j=1}^{n} H P\left(-U_{j}, 0\right) \cap C_{0}^{+}\right)=F r(Z N N)
$$

Note 8.02

$$
\begin{aligned}
& H P\left(-U_{j}, 0\right) \cap C_{0}^{+} \text {is the cone } \\
& \qquad C\left(O, U_{1}, U_{2}, \ldots, U_{j-1}, U_{j+1}, \ldots, U_{n}\right)
\end{aligned}
$$

We will call it " conic wall of non-negativity $j$ Noted $\mathrm{MCNN}_{\mathrm{j}}$.

Proof :

$$
\begin{aligned}
& \operatorname{MCNN}_{j}=H P\left(-U_{j}, 0\right) \cap C_{0}{ }^{+} \\
& \text {et } Z N N=\bigcup_{j=1}^{n} M C N N_{j}
\end{aligned}
$$

In other words, the ZNN border is none other than the conic wall meeting of non-negativity.

Note further that

So

$$
\operatorname{MCNN}_{\mathrm{j}} \subset H P\left(U_{j}, 0\right)
$$

$$
M C N N_{j} \subset \operatorname{Fr}\left(Z N N_{j}\right)
$$

\subsubsection{Exit point of ZNN following $R\left(X_{0}, V\right)$}

\section{Proposition 8.06}

We assume that $\mathrm{X}_{0}$ is in $\mathrm{ZNN}$. Since $\mathrm{ZNN}$ is closed, the exit point is the point of contact or intersection between $R\left(X_{0}, V\right)$ and $\operatorname{Fr}\left(Z N N_{j}\right)$.

Proof

Let $\mathrm{Fj}$ be the point

of $\mathrm{R}\left(\mathrm{X}_{0}, \mathrm{~V}\right)$ et $\operatorname{Fr}\left(\mathrm{ZNN}_{\mathrm{j}}\right)$ which is none other than $H P\left(-U_{j}, 0\right)$,

$$
\begin{aligned}
& I_{j} \in R\left(X_{0}, V\right), \text { so } \mathrm{I}_{\mathrm{j}} \text { is the form } \\
& \mathrm{I}_{\mathrm{j}}=\mathrm{X}_{0}+\alpha_{\mathrm{j}} \mathrm{V} \quad \text { with } \alpha_{\mathrm{j}} \geq 0
\end{aligned}
$$


Similarly

$$
I \in H P\left(-U_{j}, 0\right),
$$

So we check the equation :

$$
<-U_{j}, I>=0 \quad \text { ou }<U_{j}, I_{j}>=0
$$

From where, $\begin{aligned} & <U_{j}, X_{0}, \alpha_{j} V>=0 \\ & <U_{j}, X_{0}>+\alpha_{j}<U_{j}, V>=0\end{aligned}$

Finally, we obtain the equation:

$$
\alpha_{j}<U_{j}, V>=-<U_{j}, X_{0}>
$$

with the condition $\alpha_{j} \geq 0$.

Based on the results of section 5.2, we can say that the research axis $R\left(X_{0}, V\right)$ leads us outside of $\mathrm{ZNNj}$ only if $\mathrm{V}$ is oriented outside $\mathrm{ZNN}_{\mathrm{j}}$, that is, if

$$
<-U_{j}, v>>0 \text {, }
$$

in which case,

$$
\alpha_{j}=\frac{-<U_{j}, X_{0}>}{<U_{j}, V>}
$$

Let $X_{0}=\left(X_{0}^{i}\right) i=1, \ldots, n$

$$
\begin{aligned}
\mathrm{V} & =\left(\mathrm{v}_{\mathrm{i}}\right) \mathrm{i}=1, \ldots, \mathrm{n} \text { and }\left\langle\mathrm{U}_{\mathrm{j}}, \mathrm{X}_{0}>=\mathrm{x}_{\mathrm{i}}^{0}\right. \\
& <\mathrm{U}_{\mathrm{i}}, \mathrm{V}>=\mathrm{v}_{\mathrm{j}}
\end{aligned}
$$

The expression of $\alpha_{j}$ becomes :

$$
\alpha_{\mathrm{j}}=\frac{-\mathrm{x}_{\mathrm{i}}^{\mathrm{o}}}{\mathrm{v}_{\mathrm{j}}}
$$

Which is good

because $x_{i}^{o} \geq 0$ and $v_{j}<0$.

8.4.3 ZNN exit point following $R\left(X_{0}, V\right)$

We assume that $X_{0}$ is in ZNN. Still based on previous results, $R\left(X_{0}, V\right)$ leads us out of at least one $\mathrm{ZNN}_{\mathrm{j}}$, that is, there exists a j such that $v_{j}<0$ noting :

$$
V=\left(v_{j}\right) j=1, \ldots, n \text {. }
$$

Let IN (V) denote the set of indices $\mathrm{j}$ such that $v_{j}<0$.

$$
I N(V)=\left\{j \in[1, n] \text { tel que } v_{j}<0\right\}
$$

Hence the proposal :

\section{Proposition 8.07}

The research axis is in the non-negativity zone ZNN only if IN (V) is non-empty. In this case, the exit point $\mathrm{I}$ is given by :

$$
I=X_{0}+\alpha V
$$

Or

$$
\begin{gathered}
\alpha=\min \left\{\frac{-x_{i}^{o}}{v_{j}}\right\} \\
j \in \operatorname{In}(V)
\end{gathered}
$$

Proof:

Given that $R\left(X_{0}, V\right)$ is a totally ordered set, the exit point of I such that verify:

$$
\begin{aligned}
& I=\min \left(I_{j}\right) \\
& \quad j \in I N(V) \quad \text { where } I_{j}=X_{0}\left(X_{i}^{0}\right) V
\end{aligned}
$$

The Proof is immediate. The output point $\mathrm{I}$ is none other than the first smallest $\mathrm{I}_{\mathrm{j}_{\text {. }}}$

\section{CONCLUSION}

Through this article, we presented all the art mathematical object classes required for topo-geometric modeling MZ and the mean objectives associated with Topo-geometric definitions, as well as the conventional operations and properties of constraints LPP defining hyperplanes, are mentioned.

\section{REFERENCES}

[1] Zionts, S 1965 "Size reduction technology of linear programming and Their Applications", PhD Thesis, Carnegie Institute of Technology.

[2] T. Gal, "Weakly redundant constraints and their impact on optimal post analysis," European journal of operational R FSS, vol. 60, pp. 315-326 1979.

[3] G. Brearley, G. Mitra, HP and Williams, "Analysis of mathematical programming problems prior to Applying the simplex algorithm," Mathe matical Programming, Vol. 8, pp. 54-83, 1975.

[4] N.V. Stojkovic and PS Stanimirovic, "Two Direct methods in linear programming," 'European Journal of Operational Research, Vol. 131, no. 2, pp. 417-439 2001.

[5] J. Telgen, "Identifying redundant constraints and implicit Equalities in system of linear constraints," Management Science, Vol. 29, no. 10, pp. 1209-1222, 1983.

[6] T.Gal, "Weakly redundant constraints and their impact on optimal post analysis," European Journal of Operational Research, vol. 60, pp. 315-326 1979.

[7] S._Paulraj, P. Sumathi, "A Comparative Study of Redundant constraints Identification Methods in Linear Programming Problems ", Mathematical Problems in Engineering, Hindawi Publishing Corporation, Article ID 723402, 2. 